\title{
VARIABILIDADE ESPACIAL DE CARACTERÍSTICAS DE SOLOS NA REGIÃO DO PLANALTO MÉ DIO, RS: II. ANÁLISE DA SEMIVARIÂNCIA E DA VARIÂNCIA ${ }^{(1)}$
}

\author{
M.v.d. BERG ${ }^{(2)} \&$ E. KLAMT $^{(3)}$
}

\begin{abstract}
RESUMO
A variabilidade espacial das características de solos da região do Planalto Médio (RS) foi estudada em seis glebas cultivadas com soja, por meio das análises da semivariância e da variância, em amostragem sistemática, para confirmar e detal har resultados obtidos por análise da variância por amostragem aninhada; estimar a variância nas características dentro de unidades de mapeamento de solos e a extensão do uso de mapas de solos para avaliação de terras. 0 trabalho de campo foi realizado de dezembro de 1989 a março de 1990. Os resultados confirmam que características de solos dependentes de processos pedogenéticos mostram interdependência espacial de $500 \mathrm{~m}$ ou mais, porém a variância de características afetadas pelo manejo persistirá em espaçamentos menores. Outrossim, que qualidades da terra, como disponi bilidade de nutrientes, não podem ser extraídas apenas de mapas de solos e que métodos alternativos de amostragem devem ser testados para amenizar o efeito negativo da variância de características a curtas distâncias na qualidade de mapas pedológicos.
\end{abstract}

Termos de indexação: amostragem sistemática, análise da semivariância, caracterização de solos, qualidade de terras.

\section{SUMMARY: SPATIAL VARIABILITY OF SOIL CHARACTERISTICS IN THE PLANALTO MÉDIO REGION, STATE OF RIO GRANDE DO SUL, BRAZIL: II. ANALYSIS OF SEMIVARIANCE AND ANALYSIS OF VARIANCE}

The spatial variability of soil characteristics in the Planalto Médio region, State of Rio Grande do Sul, Brazil, was studied in six areas cultivated with soybean. Semi variance and varianceanalyses wereapplied to the data obtained by systematic soil sampling to confirm and

\footnotetext{
(1) Parte da tese de doutorado do primeiro autor, a ser apresentada na Utrecht University, Holanda. Trabalho apresentado no XXV Congresso Brasileiro de Ciência do Solo, em julho 1995. Viçosa, MG. Recebido para publicação em fevereiro de 1996 e aprovado em março de 1997.

(2) Professor Assistente I, Faculdade de Agronomia e Engenharia Florestal, Universidade Eduardo Mondlane, Maputo, Moçambique.

(3) Professor Visitante da UFSM eaposentado do Departamento de Solos, Faculdade deAgronomia/UFRGS. Caixa Postal 776, CEP $90001-970$ - Porto Alegre (RS). Bolsista do CNPq.
} 


\begin{abstract}
detail results obtained by nested analysis of variance, and to estimate the variance of characteristics within soil mapping units and therange of its application for land evaluation. Field work was performed from December, 1989 to March, 1990. Theresul ts confirmed that soil characteristics dependent on pedogenetic processes showed spatial interdependenceup to $500 \mathrm{~m}$ or more, but the variance of characteristics affected by soil management would persist within shorter distances. Furthermore, the results indicated that land qualities such as nutrient availability cannot be extracted only from soil maps and that al ternativemethods of sampling and evaluation of spatial variability at short distances should beinvestigated to minimize the negative effect on the quality of soil maps.
\end{abstract}

Index terms: systematic sampling, semivariance analysis, soil characterization, Iand quality.

\section{INTRODUÇÃO}

A variabilidade das características dos solos constitui um problema, porque, em muitos casos, não éaparente e porque a maioria das características não pode ser medida continuamente no espaço. Burrough (1983) apresentou diversos exemplos de como o mapeamento de sol os com crescentes detal hes revela novas estruturas de variação espacial.

A literatura sobre variabilidade espacial de solos fortemente intemperizados, de argila de atividade baixa, é escassa. No Brasil, a preocupação sobre a variabilidade do sol o dentro de unidades cartográficas já foi expressa nos anos 70s, em trabal hos que usaram a estatística clássica, como os de Oliveira \& Rotta (1973), Oliveira (1975) e Cadima et al. (1980), todos efetuados no Estado de São Paulo. Os relatórios de levantamentos de solos do Instituto Agronômico de Campinas (por exemplo, Oliveira et al., 1982) apresentaram tabelas com dados de variação das princi pais características e propriedades variáveis de solos em cada unidade de mapeamento. Em geral, dentro dessas unidades de mapeamento, pode-se notar uma variabilidade considerável das características químicas, como saturação por bases e soma de bases trocáveis, enquanto as uni dades são mais homogêneas para características como textura e matéria orgânica. Não se pode excluir a possibilidade de parcialidades nestes resultados, pois as análises estatísticas são efetuadas para as mesmas observações anteri ormente usadas para delinear as unidades de mapeamento. Também não é possível fazer nenhuma afirmação a partir dessas informações sobre os padrões de variação espacial das características dos solos dentro das unidades de mapeamento.

Os trabalhos consultados que usaram a teoria das variáveis regionalizadas no Brasil referem-setodos a escalas espaciais pequenas, de menos que $1 \mathrm{~m}$, até pouco mais que $100 \mathrm{~m}$. A variabilidade espacial nessas escalas tem maiores implicações para a pesquisa de campo. Nestes estudos, encontrou-se, geralmente, a mai or parte da variação dos solos no intervalo de 5 a $15 \mathrm{~m}$ de distância. Por exemplo, Bacchi, mencionado por Reichardt et al. (1986), amostrou, com espaçamento de $1 \mathrm{~m}$, um latossolo vermel ho-escuro orto sob canade-açúcar em Araras (SP), numa transeção de $50 \mathrm{~m}$. Os valores de autocorrelação para pH foram significativos (nível de $5 \%$ ) até para 5 m dedistância. $\mathrm{O}$ desvio-padrão do $\mathrm{pH}$ foi de 0,35 , com valores extremos de 4,6 e6,3. Libardi et al . (1986) analisaram a variação da umidade, da textura e da densidade de partículas ao longo de uma transeção de $150 \mathrm{~m}$, em distâncias regulares de 0,5 m, numa terra roxa estruturada em Piracicaba (SP). Todas as variáveis, exceto silte, mostraram dependência espacial até, aproximadamente, $15 \mathrm{~m}$. No silte, ela foi de $40 \mathrm{~m}$. É interessantenotar queas formas dos semivariogramas para as diferentes variáveis foram bastantediferentes, sugerindo variação cíclica para a densidade das partículas. Os desvios-padrão para textura foram pequenos: $40 \mathrm{~g} \mathrm{~kg}^{-1}$ para argila, $20 \mathrm{~g} \mathrm{~kg}^{-1}$ para areia e $30 \mathrm{~g} \mathrm{~kg}^{-1}$ para silte. Um estudo da variabilidade da profundidade e espessura da compactação do sol o na mesma área (Silva et al., 1989), ao longo de uma transeção de $40 \mathrm{~m}$, com observações a cada $10 \mathrm{~cm}$, mostrou que as distâncias nas quais as amostras são consideradas dependentes corresponderam a menos de $2 \mathrm{~m}$. A presença de variações cíclicas, neste caso, foi atribuída ao manejo do solo.

Para efetuar levantamentos pedológicos, porém, é necessário quantificar a variabilidade espacial em escalas de centenas de metros até quilômetros. Burrough (1991), entre outros, propôs metodologia para a determinação da variabilidade de características de solo facilmente mensuráveis em grande densidadee sua correlação com variáveis de obtenção mais onerosa, observadas em densidade menor. Berg \& Klamt (1997), com base na hipótese que pode existir uma analogia de variâncias espaciais em regiões diferentes, mas com solos similares, estudaram a variabilidade espacial de características de solos em sete gl ebas na região do Planalto Médio (RS), por meio da análise da variância por amostragem aninhada. Esse estudo mostrou que amostragens sistemáticas em grades, com pontos distanciados de $600 \mathrm{~m}$, é suficiente para pesquisar a variabilidade das principais características dos solos.

O presente trabal ho tem como objetivo detal har e confirmar os resultados obtidos por Berg\& Klamt (1997), por meio da amostragem sistemática e utilização da análise da semivariância e da variância por gleba. Mais especificamente, objetiva-se determinar: (1) a maneira como sol os intensamente intemperizados na região do Planal to M édio variam espacial mente; (2) a extensão em que mapas de solos podem ser usados para procedimentos de avaliação de terras na região, e(3) a variância mínima esperada dentro de unidades de mapeamento em mapas a serem produzidos. 


\section{MATERIAL E MÉTODOS}

\section{Amostragem de campo}

As glebas amostradas no presente estudo correspondem às de números 1 a 6 da amostragem aninhada efetuada na região do Planalto Médio por Berg \& Klamt (1997). Todas as gl ebas possuíam uma área aproximada de 60 ha e estavam cultivadas com soja em sistema de manejo avançado; eram relativamentehomogêneas, conformeindicava o mapa de solos e os aspectos fisiográficos, bem como os resultados da análise da variância por amostragem aninhada.

As glebas foram amostradas de tal maneira que pontos de observação vizinhos estavam afastados entre 250 e $300 \mathrm{~m}$, o que resultou em 10 a 21 pontos de amostragem por gl eba. Os pontos de amostragem não consistiram em uma grade totalmente regular, em vista do contorno irregular das glebas. Os pontos foram locados em mapas antes da coleta no campo, evitando, assim, parcialidade e assegurando uma distribuição regular. A amostragem foi sempre feita entre as linhas das culturas, quando presentes. Amostraram-se 97 pontos na profundidade de 0-20 e $60-80 \mathrm{~cm}$.

A localização geográfica em coordenadas UTM dos pontos foi determinada por meio de mapas planialtimétricos, em escala 1:50.000, produzidos, em 1979, pelo Ministério do Exército e complementados por mapas com escala de 1:5.000 e 1:10.000, fornecidos pel os agricultores.

No campo, estimaram-se os ângulos de dedive $\left(\mathrm{cm} \mathrm{m}^{-1}\right)$ com um clinômetro de bol so e determinou-se a cor das amostras de terra (Escala Munsell). Os matizes das cores foram transformados para valores numéricos, conforme Lepsch et al. (1978).

\section{Métodos de laboratório}

As análises físicas e químicas das amostras foram efetuadas nos laboratórios de Pedol ogia eFertilidade do Instituto Agronômico de Campinas (IAC)/(SP). Os métodos usados estão de acordo com os descritos por Camargo et al. (1986), exceto para fósforo, que foi determinado segundo Raij \& Quaggio (1983). As seguintes características dos sol os foram analisadas: concentração de argila ( $<2 \mu \mathrm{m})$, pelo método de pi peta e dispersão com hexametafosfato de sódio e $\mathrm{NaOH}$; pH em solução de $\mathrm{KCl} 1 \mathrm{~mol} \mathrm{~L}^{-1}(1: 2,5)$; carbono orgânico (C), pela oxidação com bicromato de potássio (Walkley-Black); fósforo disponível $\left(\mathrm{P}, \mathrm{mg} \mathrm{kg}^{-1}\right)$, pela extração com a resina trocadora de íons; soma das bases trocáveis $\mathrm{S}\left(\mathrm{Ca}+\mathrm{Mg}+\mathrm{K}+\mathrm{Na}\right.$; $\left.\mathrm{mmol}_{\mathrm{C}} \mathrm{kg}^{-1}\right)$ extraídas com $\mathrm{NH}_{4} \mathrm{OAC} 1 \mathrm{~mol} \mathrm{~L}^{-1} \mathrm{em} \mathrm{pH}$ 7. A capacidade de troca catiônica $\left(C T C, \mathrm{mmol}_{\mathrm{C}} \mathrm{kg}^{-1}\right)$ foi cal culada pela soma de $\mathrm{S}$, mais a acidez potencial $\left(\mathrm{H}+\mathrm{Al}, \mathrm{mmol}_{\mathrm{c}} \mathrm{kg}^{-1}\right)$ extraída com acetato de cálcio $1 \mathrm{~mol} \mathrm{~L}^{-1} \mathrm{em} \mathrm{pH} 7$ etitulação com $\mathrm{NaOH}, 0,1 \mathrm{~mol} \mathrm{~L}^{-1}$. A percentagem de saturação por alumínio $(\mathrm{m})$ foi calculada por $100 \% * \mathrm{Al} /(\mathrm{S}+\mathrm{Al})$, onde Al é a acidez trocável $\left(\mathrm{mmol}_{\mathrm{c}} \mathrm{kg}^{-1}\right)$ extraída com $1 \mathrm{~mol} \mathrm{~L}-1 \mathrm{KCl}$ e titulação com $\mathrm{NaOH}$ 0,1 mol L-1. A percentagem de saturação por bases (V) foi cal culada por 100 (S/CTC).

\section{Análise estatística}

Os cálculos de média, variância, assimetria e curtose e a análise de variância dentro e entre gl ebas foram feitos conforme Wilkinson (1992), enquanto a análise de semivariância foi adaptada de Burrough \& Keulen (1987). O variograma (g) foi determinado em função de $I_{\text {lag, }}$ de todos os pares com distância mútua entre $\left(l_{\operatorname{lag}}-1\right) * d$ e $1_{\operatorname{lag}} * d$, onde $l_{\text {lag }}$ é um valor inteiro que variou desde 1 atéo valor máximo definido. Para d, foi usado o val or de 0,3 km. I sso significa que, para $\mathrm{I}_{\mathrm{Iag}}=1$, todos os pares com distância mútua entre 0,0 e 0,3 km foram considerados; para $\mathrm{I}_{\mathrm{lag}}=2$, todos os pares de dados entre 0,3 e 0,6 km, etc.

\section{RESULTADOS E DISCUSSÃO}

\section{Estatística geral}

É apresentada na parte superior dos quadros $1 \mathrm{e}$ 2 , respectivamente, para as profundidades de amostragem de $0-20$ e de $60-80 \mathrm{~cm}$. Os dados indicam que a concentração de argila e a saturação por alumíniosão, consideravel mente, maiores na camada de $60-80 \mathrm{~cm}$ do que na superficial. J á $\mathrm{pH}, \mathrm{V}, \mathrm{P}, \mathrm{S}$ e CTC sãomaiores nos $20 \mathrm{~cm}$ superficiais. A maior parte das diferenças das características químicas entre as camadas de $0-20 \mathrm{~cm}$ e $60-80 \mathrm{~cm}$ são resultantes de calagem e fertilização, que afetaram em maior grau a camada de $0-20 \mathrm{~cm}$. A maior CTC nessa camada explica-setambém pelo teor de matéria orgânica mais el evado no horizonte superficial.A presença de teores mais baixos de argila na camada de $0-20 \mathrm{~cm}$ pode refletir a influência do material deorigem na formação do solo, bem como de certa migração de argila para o horizonte B.

\section{Análise da semivariância}

Os resultados da análise da semivariância (Quadros 1 e 2) indicam que, para todas as variáveis, a maior parte da variância nas glebas ocorre em distâncias menores do que 300 m. Para a maioria das variáveis da camada de 60-80 cmeal gumas de 0-20 cm, não foi possível detectar nenhuma estrutura espacial da variância, para os intervalos anal isados. Os valores de $\gamma$ para o intervalo de $1,4 \mathrm{~km}$ e, provavelmente, também os para o de 1,0 km, devem ser analisados com cautela, porque, embora o número de pares de dados pareça suficiente, o número de observações realmente independentes é pouco superior ao das glebas, em vista de os pares de dados estarem próximos um do outro. Isso resulta em alguns semivariogramas peculiares, como os para $\mathrm{m}$ e $\mathrm{P}$ da camada de $0-20 \mathrm{~cm}$, os quais apresentam uma semivariância que diminui com o aumento dos intervalos, e os de S eV da camada de $60-80 \mathrm{~cm}$, com mudanças abruptas. As diferenças entre valores de $\gamma$ 
para os intervalos de 0,3 e 0,6 km são, geralmente, pequenas.

\section{Análise da variância por gleba}

Os resultados da análise da variância por gleba encontram-se também nos quadros 1 e 2 . Mais de $85 \%$ da variância total doteor deargila na camada de 0-20 cm é explicada pela análise da variância, bem como outras características de solo, com exceção do croma e da concentração em P. As diferenças em variância na camada de $0-20 \mathrm{~cm}$ para a de $60-80 \mathrm{~cm}$ são, provavelmente, causadas pelo manejo, pois a aplicação de cal cário causou a di minuição deAl trocável e a saturação por $\mathrm{Al}(\mathrm{m})$ da camada de $0-20 \mathrm{~cm}$, tornando as glebas mais similares. $\mathrm{No}$ caso de $\mathrm{pH}$, a variância entre glebas é maior para a de $0-20 \mathrm{~cm}$, provavel mente devido a quantidades diferentes e/ou à distribui ção não homogênea dos corretivos aplicados. Nesses solos com CTC muito baixa, $m$ decresce até praticamente zero, sem relação com a quantidade de calcário aplicado, acima de um valor crítico, que corresponde a $\mathrm{pH}$ 5,5. O resultado é uma variância grande do $\mathrm{pH}$ e pequena de $\mathrm{m}$ na camada superficial. $A$ variância de $P$ também é muito el evada nessa camada, provavelmente, induzida pela aplicação heterogênea de $\mathrm{P}$ nas fertilizações. No entanto, para a mai oria das variáveis, a análiseda variância explicou parte menor da variância das características da camada de 60-80 cm doque das características de 0-20 cm. A causa disso não é uma heterogenei dade da camada subsuperficial, mas, sim, a uniformidade geral das suas características, em todas as glebas. Para a maioria das características da camada de $60-80 \mathrm{~cm}$, a variância ponderada dentro das glebas é menor do que na de $0-20 \mathrm{~cm}$.

\section{E feito da transformação logarítmica dos dados}

Em alguns casos, verificou-se que a assimetria ea curtose dos dados originais e dos resíduos da análise de variância apresentaram desvios de zero (Quadros 1 e 2) quando o somatório desses valores deveria ser zero. A análise da semivariância de dados transformados e a assimetria - curtose dos resíduos transformados (log) (Quadros 1 e 2) mostram que variáveis com distribuição fortemente assimétricas ou cúrticas (notadamente para P) obtêm uma distribuição mais próxima da normal após a transformação logarítmica. Essa transformação também ajuda a tornar dados distantes menos importantes, ea variância nas glebas mais homogênea. A transformação logarítmica também dei xou as diferenças entreas camadas menos evidentes. A transformação pareceu mais apropriada para S, m\% $(0-20 \mathrm{~cm})$ e V $(60-80 \mathrm{~cm})$. A variância determinada pela análise da variância não mudou muito para a maioria das características, mas, em alguns casos, a transformação levou a valores, consideravel mente, diferentes. As mudanças não são consistentes. Por exemplo, 53\% da variância em m na camada de $0-20 \mathrm{~cm}$ foi determinada pela análise da variância. Essa percentagem aumentou para 69 após a transformação. No caso da mesma característica, porém na camada de $60-80 \mathrm{~cm}$, a percentagem da variância, determinada pela análise da variância, diminuiu, após a transformação, de 47 para $25 \%$.

\section{Comparação de variância aninhada com semivariância}

Os resultados apresentados revelam discrepâncias entre as análises da semivariância e a da variância por gleba e por amostragem aninhada. No caso da concentração em argila, a variância dentro de gl ebas é um pouco maior nos resultados das análises da amostragem geral (semivariância, análise da variância) do que na amostragem aninhada, verificada por Berg \& Klamt (1997). A variância total da concentração em argila na camada de $60-80 \mathrm{~cm}$, na amostragem geral, também é maior do que na aninhada. Para o matiz da cor, a análise aninhada indicou valores maiores de variância para a camada de 60-80 cm do que para a superficial, para o intervalo deamostragem mais el evado (entre glebas). A análise da variância para as amostras de cobertura geral sugeriu o inverso.

A mai oria das discrepâncias podeser explicada pelo número limitado de observações, principalmente na análise aninhada usada por Berg \& Klamt (1997). É necessário considerar também que alguns métodos usados foram diferentes, como, por exemplo, teor de argila deter minado ao tato na amostragem aninhada vérsus método da pipeta no presente estudo.

No entanto, em geral, os variogramas aninhados concordam bem com os semivariogramas que se baseiam num esquema de amostragem muito mais intensivo. A análise aninhada permitiu, de maneira rápida, uma indicação aproximada dos padrões espaciais, que podem servir como base para a determinação da densidade de amostragem. A amostragem aninhada não permite a obtenção de informações mais detal hadas sobre a variabilidade espacial, enquanto o número de amostras for pequeno. Caso uma amostragem maior seja adotada, deve-se preferi-la com densidade mais homogênea ou em transeções. Corsten \& Stein (1991) chegaram a uma conclusão similar, quando compararam os resultados de interpolação para diferentes quadros de amostragem.

\section{Comparação da semivariância com a análise da variância por gleba}

Teoricamente, o patamar da semivariância deveria ser igual à variância dentro das gl ebas. Para a maioria das características, a variância dentro das gl ebas está muito perto da semivariância nos interval os entre 0,3 e 0,6 km. I sso não significa, necessariamente, que o alcance da dependência espacial está entre 0,3 e $0,6 \mathrm{~km}$, porqueas gl ebas têm uma superfície restrita. $\mathrm{Na}$ maioria dos casos, a maior parte da variância dentro das glebas já estava presente no intervalo de $0,3 \mathrm{~km}$. I sso sugere que se ganha pouco pelo uso de métodos de interpolação, que consideram a dependência espacial. Aparentemente, as glebas podem, igual mente, ser descritas em termos clássicos de média e variância. 


\section{Otimização da amostragem}

Na região de estudo, existem dois níveis principais de variabilidade das características dos sol os. Aquela relacionada com o material de origem, equenão pode ser modificada com facilidade, como por exemplo, teor de argila e CTC, que podem ser determinados numa densidade de amostragem de, aproximadamente, uma observação por quilômetro quadrado.

As modificações impostas pelo manejo resultam numa variabilidade considerável, a curto espaço, notadamente para o P na camada de $0-20 \mathrm{~cm}$, decorrente da fertilização, e para $\mathrm{V}$ e pH , decorrente da calagem. O contrário observa-se com $\mathrm{m} \%$, cuja variância diminuiu em todos os níveis, devido à diminuição geral dos níveis de Al-trocável nos solos onde foi aplicado o cal cário. A variabilidade espacial de alcance intermediário foi de importância menor. Essa variabilidade está, possivelmente, relacionada com a fisiografia da área (J ansen, 1991). A variabilidade espacial que ocorreu em distâncias pequenas praticamente não pode ser determinada. A amostragem composta talvez poderia amenizar as variações a curtas distâncias e filtrar aquelas com alcance médio, as quais poderiam ser mapeadas sem custos adicionais excessivos. Essas amostras

Quadro 1. Sumário de análises estatísticas das características dos solos estudados em amostragem sistemática em seis glebas, profundidade de $0-20 \mathrm{~cm}$, no Planalto Médio (RS) ${ }^{(1)}$

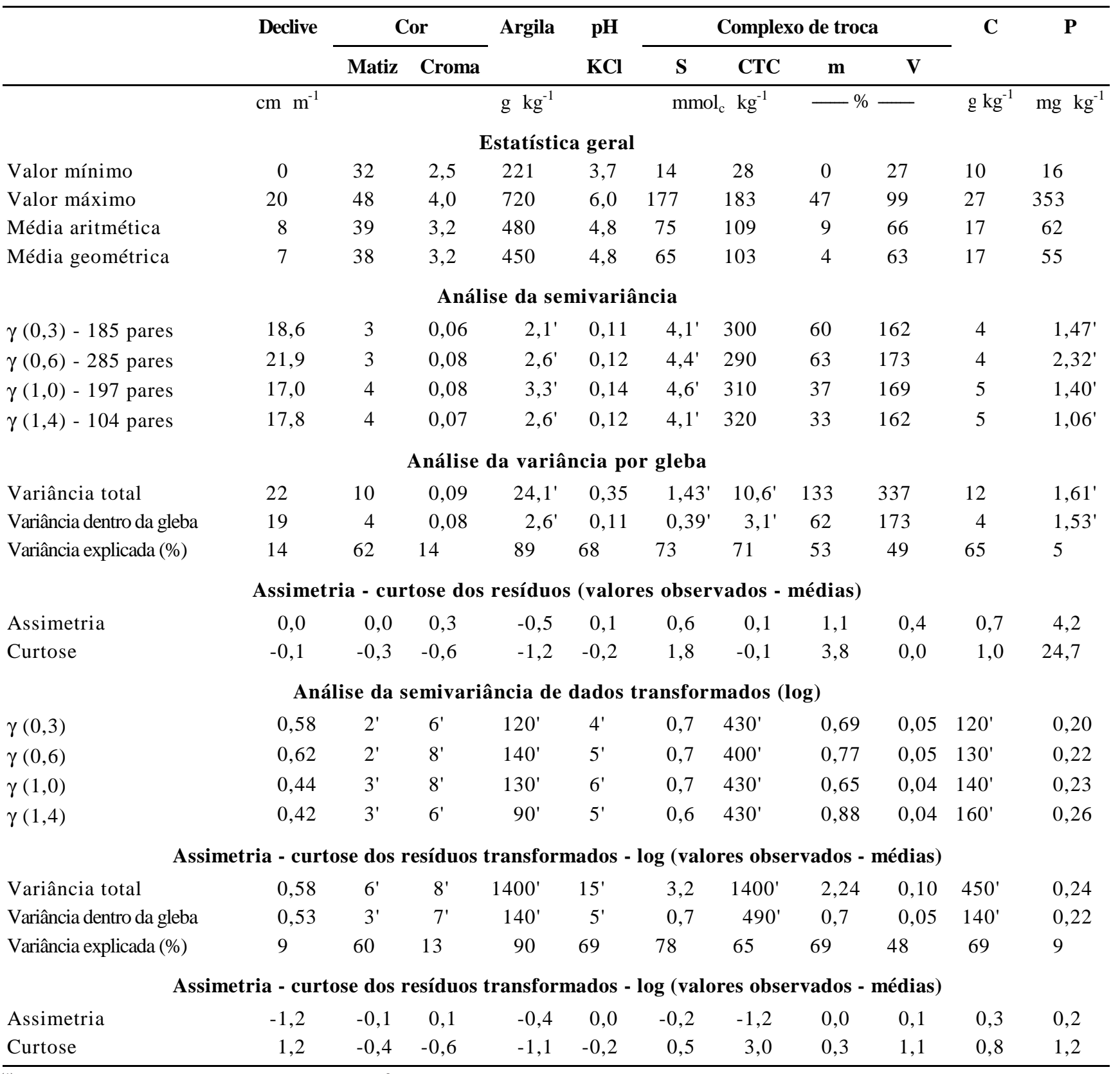

\footnotetext{
(1) Multiplicar valores assinalados com ' por $10^{3}$.
} 
compostas deveriam consistir em amostras coletadas dentro de 1 a $5 \mathrm{~m}$.

\section{Conseqüências para a avaliação das terras}

Os resultados indicaram que a disponibilidade de nutrientes, que é de importância especial para a produção agrícola, não pode ser inferida somente de mapas de solos. Relatórios da história do manejo das glebas podem ajudar a determinar essa qualidade de terras. E m regiões nunca utilizadas para a agricultura, os solos tendem a apresentar maior variabilidade sistemática do que ao acaso (U pchurch \& Edmonds, 1991) e, por conseguinte, os mapas de solos podem refletir melhor a variabilidade espacial das características químicas dos solos, do que no presente estudo.

No caso da utilização de modelos de rendimento de culturas para a avaliação das terras, fica claro que alimentar os modelos apenas com dados de perfis representativos pode levar a uma propagação de erro pel o fato de não levar em conta as variações induzidas pelo manejo das áreas, conseqüentemente, de curta distância, sem considerar o fato de os perfis caracterizados nos levantamentos de sol os não serem representativos para as unidades de mapeamento delineadas.

\section{Comparação com estudos anteriores}

Os resultados deste estudo comprovam aqueles já publicados e referidos. Este estudo também mostrou uma variabilidade relativamente grande a curta distância, para as características facilmente modificadas pelo manejo $(\mathrm{P}, \mathrm{pH}, \mathrm{V})$ da camada superficial, enquanto outras características, como o teor de argila e de C e a CTC se mostraram homogêneas nas glebas, em comparação com a variabilidade total na região. É importante notar que este estudo cobriu mais níveis espaciais que as publicações

Quadro 2. Sumário de análises estatísticas das características dos solos estudados em amostragem sistemática em seis glebas, profundidade de $60-80 \mathrm{~cm}$, no Planalto Médio (RS)(1)

\begin{tabular}{|c|c|c|c|c|c|c|c|c|c|c|}
\hline & \multicolumn{2}{|c|}{ Cor } & \multirow[t]{2}{*}{ Argila } & \multirow{2}{*}{$\begin{array}{c}\text { pH } \\
\text { KCl }\end{array}$} & \multicolumn{4}{|c|}{ Complexo de troca } & \multirow[t]{2}{*}{$\mathbf{C}$} & \multirow[t]{2}{*}{$\mathbf{P}$} \\
\hline & Matiz & Croma & & & $\mathbf{S}$ & CTC & $\mathbf{m}$ & $\mathbf{V}$ & & \\
\hline & & & \multicolumn{2}{|l|}{$\mathrm{g} \mathrm{kg}^{-1}$} & \multicolumn{2}{|c|}{$-\mathrm{mmol}_{\mathrm{c}} \mathrm{kg}^{-1}-$} & \multicolumn{2}{|c|}{$-\%-$} & $\mathrm{g} \mathrm{kg}^{-1}$ & $\mathrm{mg} \mathrm{kg}^{-1}$ \\
\hline \multicolumn{11}{|c|}{ Estatística geral } \\
\hline Valor mínimo & 30 & 3,5 & 380 & 3,8 & 5 & 54 & 3 & 8 & 4 & 1 \\
\hline Valor máximo & 46 & 6,0 & 820 & 4,9 & 68 & 115 & 89 & 73 & 11 & 11 \\
\hline Média aritmética & 33 & 5,2 & 650 & 4,2 & 23 & 75 & 51 & 30 & 8 & 3 \\
\hline Média geométrica & 33 & 5,2 & 630 & 4,2 & 20 & 74 & 43 & 27 & 8 & 3 \\
\hline \multicolumn{11}{|c|}{ Análise da semivariância } \\
\hline$\gamma(0,3)-185$ pares & 2 & 0,24 & $1,9^{\prime}$ & 0,02 & 120 & 90 & 198 & 110 & $2,1^{\prime}$ & 2 \\
\hline$\gamma(0,6)-285$ pares & 2 & 0,22 & $2,7^{\prime}$ & 0,03 & 110 & 90 & 229 & 121 & $2,3^{\prime}$ & 2 \\
\hline$\gamma(1,0)-197$ pares & 3 & 0,22 & $2,4^{\prime}$ & 0,04 & 150 & 110 & 267 & 152 & $2,7^{\prime}$ & 2 \\
\hline$\gamma(1,4)-104$ pares & 3 & 0,15 & $2,1^{\prime}$ & 0,04 & 170 & 130 & 294 & 171 & $2,9^{\prime}$ & 4 \\
\hline \multicolumn{11}{|c|}{ Análise da variância por gleba } \\
\hline Variância total & 7 & 0,56 & $17,9^{\prime}$ & 0,04 & 170 & 120 & 422 & 195 & $2,3^{\prime}$ & 2 \\
\hline Variância dentro da gleba & 3 & 0,23 & $2,6^{\prime}$ & 0,03 & 120 & 100 & 225 & 125 & $2,3^{\prime}$ & 2 \\
\hline Variância explicada (\%) & 60 & 59 & 86 & 36 & 26 & 16 & 47 & 36 & 0 & 7 \\
\hline \multicolumn{11}{|c|}{ Assimetria - curtose dos resíduos (valores observados - médias) } \\
\hline Assimetria & 1,3 & $-0,1$ & $-0,8$ & 1,9 & 1,0 & 0,8 & $-0,8$ & 1,1 & 0 & 2,2 \\
\hline Curtose & 6,8 & $-0,7$ & 1,4 & 5,4 & 3,2 & 1,5 & 0,2 & 1,1 & $-0,5$ & 6,9 \\
\hline \multicolumn{11}{|c|}{ Análise da semivariância de dados transformados (log) } \\
\hline$\gamma(0.3)$ & $2^{\prime}$ & $11^{\prime}$ & $60^{\prime}$ & $1^{\prime}$ & 1,5 & 160 & 0,34 & 0,09 & $340^{\prime}$ & 0,12 \\
\hline$\gamma(0.6)$ & $2^{\prime}$ & $9^{\prime}$ & $80^{\prime}$ & $1^{\prime}$ & 1,7 & 160 & 0,36 & 0,12 & $370^{\prime}$ & 0,11 \\
\hline$\gamma(1.0)$ & $2^{\prime}$ & $8^{\prime}$ & $60^{\prime}$ & $2^{\prime}$ & 1,9 & 190 & 0,47 & 0,12 & $440^{\prime}$ & 0,14 \\
\hline$\gamma(1.4)$ & $2^{\prime}$ & $5^{\prime}$ & $50^{\prime}$ & $2^{\prime}$ & 2,0 & 220 & 0,54 & 0,12 & $540^{\prime}$ & 0,20 \\
\hline \multicolumn{11}{|c|}{ Assimetria - curtose dos resíduos transformados - log (valores observados - médias) } \\
\hline Variância explicada (\%) & 63 & 60 & 84 & 38 & 32 & 16 & 25 & 50 & 0 & 9 \\
\hline \multicolumn{11}{|c|}{ Assimetria - curtose dos resíduos transformados - log (valores observados - médias) } \\
\hline Assimetria & 0,9 & $-0,1$ & $-0,9$ & 1,2 & 0,2 & 0,1 & $-2,0$ & 0,3 & $-0,5$ & 0,4 \\
\hline Curtose & 4,0 & $-0,4$ & 2,0 & 1,0 & 0,6 & 0 & 4,4 & $-0,1$ & 0,4 & 1,0 \\
\hline
\end{tabular}

(1) Multiplicar valores assinalados com ' por $10^{3}$. 
consultadas, que focalizaram em distâncias muito curtas ou que usaram a estatística clássica para estudar a variância dentro de unidades de mapeamento. Estudos adicionais serão necessários para verificar se as tendências para os intervalos entre 50$1.000 \mathrm{~m}$ encontrados também se manifestam em outras regiões, com solos similares.

\section{CONCLUSÕES}

1. Uma densidade de uma amostra por $0,25 \mathrm{~km}^{2}$ ou mais (intervalo de 500-1.000 m entre pontos vizinhos) é suficiente para determinar os principais padrões espaciais dos solos fortemente intemperizados, encontrados na área de estudo. A variância de algumas características químicas dos solos, dentro das unidades de mapeamento a serem definidas, ainda será considerável. Aumentando a densidade de amostragem para uma por 0,0025 km² (isto é, intervalos de $50 \mathrm{~m}$ ) resultaria em reduzidos melhoramentos da qualidade do levantamento dos solos;

2. Métodos de amostragem alternativos, como a amostragem composta, devem ser estudados para determinar se as variações a distâncias muito pequenas (<10 $\mathrm{m}$ ) podem ser avaliadas e definidas;

3. A disponibilidade de nutrientes não pode ser inferida apenas a partir de mapas de solos, porque é muito influenciada pelo manejo;

4. Os sistemas de classificação de solos existentes e legendas de mapas de sol os não levam em conta que a agricultura intensiva pode modificar, facilmente, algumas características dos solos. Assim, não é aconsel hável usar atributos, como saturação por bases do horizonte superficial, como critério diagnóstico para a dassificação dos solos em áreas com níveis avançados de manejo, ou horizontes diagnósticos superficiais definidos com base nesse atributo.

\section{AGRADECIMENTOS}

Os autores agradecem à Fundação de Amparo à Pesquisa do Rio Grande do Sul (FAPERGS) e à Fundação de Amparo à Pesquisa Científica nos Trópicos (WOTRO), Holanda, os recursos recebidos; à Faculdade de Agronomia da Universidade de Passo Fundo e ao Centro Nacional de Pesquisa do TrigoCNPt/EMBRAPA, o apoio; ao Instituto Agronômico de Campinas (SP), a realização das análi ises de solo, e aos proprietários rurais, Srs. Bertagnolli, Grazziotin, Augustin e Marinho, o acesso às propriedades, informações referentes ao manejo dos solos e rendimentos obtidos e apoio, o que tornou esta pesquisa possível.

\section{LITERATURA CITADA}

BERG, M. van den \& KLAMT, E. Variabilidade espacial de características de solos na região do Planalto Médio, RS: I. Análise da variância por amostragem aninhada. R. bras. Ci. Solo, Viçosa, 21:393-399, 1997.

BURROUGH, P.A. Multiscale sources of spatial variation in soils. I. The application of fractal concepts to nested levels of soil variation. J. Soil Sci., London, 34:577-597, 1983.

BURROUGH, P.A. Sampling designs for quantifying map unit composition. In: Spatial variabilities of soils and landforms. Soil Science Society of America, Madison, 1991. p.89-125 (Special Publication, 28)

BURROUGH, P.A. \& KEULEN, J. Van. PC-GEOSTAT - a set of programs for geostatistical analysis. Utrect, The Netherlands. University of Utrecht, 1987. p.123.

CADIMA, Z.A.; LIBARDI, P.L. \& REICHARDT, K. Variabilidade espacial da condutividade hidráulica em um latossolo amarelo textura média no campo. R. bras. Ci. Solo, Campinas, 4:63-66, 1980.

CAMARGO, A.O.; MONIZ, A.C.; J ORGE, J.A. \& VALADARES, J.M.A.S. Métodos de análise química, mineralógica e física de solos do Instituto Agronômico de Campinas. Campinas, IAC, 1986. 94p. (Boletim técnico, 106)

CORSTEN, L.C.A. \& STEIN, A. Are nested designs recommendable for spatial semivariogram estimation? In: STEIN, A., ed. Spacial I nterpolation. Wageningen, Agricultural University, 1991. p.41-70.

J ANSEN, L. Soil-landscape relations and a distance-price relation for sugarcane in the assis area, São Paulo State, Brazil. Wageningen, Agricultural University, 1991. p.88. (Dissertação de Mestrado)

LEPSCH, I.F.; MENK, J.R.J .; OLIVEIRA, J.B.; SILVA, O.A.L.; MASSON, W. \& BORDINI, O. Apoio computacional em levantamento de solos. Campinas, Instituto Agronômico, 1978. 44p. (Boletim, 210)

LIBARDI, P.L.; PREVEDELLO, C.L.; PAULETTO, E.A. \& MORAES, S.O. Variabilidade espacial da umidade, textura e densidade de partículas ao longo de uma transeção. R. bras. Ci. Solo, Campinas, 10:85-90, 1986.

OLIVEIRA, J.B. Diferenciação e distribuição em diversos níveis categóricos de duas áreas aparentemente homogêneas. Bragantia, Campinas, 34:310-348, 1975.

OLIVEIRA, J.B.; MENK, J .R.F.; BARBIERI, J.L.; ROTTA, C.L. \& TREMOCOLDI, W. Levantamento pedológico semidetalhado do Estado de São Paulo: quadrícula de Araras. Campinas, Instituto Agronômico, 1982. 180p. (Boletim técnico, 72)

OLIVEIRA, J .B. \& ROTTA, C.L... Apreciação generalizada sobre a variação das características químicas das unidades de solo da Estação Experimental de Limeira. Bragantia, Campinas, 32:62-69, 1973.

RAIJ , B. van \& QUAGGIO, J.A. Métodos de análise de solo para fins de fertilidade. Campinas, Instituto Agronômico, 1983. 31p. (Boletim técnico, 81) 
REICHARDT, K.; VIEIRA, S.R. \& LIBARDI, P.L. Variabilidade espacial de solos e experimentação de campo. R. bras. Ci. Solo, Campinas, 10:1-6, 1986.

SILVA, A.P. da; LIBARDI, P.L. \& VIEIRA, S.R. Variabilidade espacial da resistência à penetração de um latossolo vermel ho-escuro ao longo de uma transeção. R. bras. Ci. Solo, Campinas, 13:1-5, 1989.
UPCHURCH, D.R. \& EDMONDS, W.J . Statistical procedures for specific objectives. In: MAUSBACH, M.J . \& WILDING, L.P. eds. Spatial variabilities of soil land-forms. Madison, Soil Science Society of America, 1991. p.73-88. (Special Publication, 28)

WILKINSON, L. Systat for windows, version 5. Evanston, Systat, Inc., 1992. p.750. 